\title{
Acknowledgement to Referees
}

(c) The Author(s) 2019

\section{Dear Reader,}

This is the final issue of PharmacoEconomics - Open for 2019 and we would like to take this opportunity to reflect on the year and thank everyone who has contributed to the quality of the journal's content.

We have published 81 articles over the past 12 months, which is a significant achievement for a journal that is only in its third year of publication. We have many people to thank for this.

Firstly, we thank all of the authors who have contributed quality articles to PharmacoEconomics - Open this year. Next, we thank the many peer reviewers who have dedicated their time and effort to ensuring the quality of the journal's content. We acknowledge the following people who have reviewed for the journal in the past 12 months:

Blythe Adamson, USA

Ilke Akpinar, Canada

Luz-Ma-Adriana Balderas Peña, Mexico

Graeme Ball, Canada

Pelham Barton, UK

Mario Battaglia, Italy

Inigo Bermejo, UK

Laura Bojke, UK

Hana Broulikova, Czech Republic

Ash Bullement, UK

Thomas Butt, UK

Sean Candrilli, USA

Hannah Carter, Australia

Amanda Cole, UK

Tracy Comans, Australia

Anna Coutinho, USA

Kathryn Coyle, UK

Irina Degtiar, USA

Jacques Demotes, France

John Denny, India

Piyameth Dilokthornsakul, Thailand

Michael Eaddy, USA

Lars Ehlers, Denmark

Francis Fatoye, UK

Eli Feiring, Norway

Steven Feldman, USA

Elisabeth Fenwick, UK

Lan Gao, Australia

Jose García-Domínguez, Spain
Denis Getsios, USA

Daniel Geynisman, USA

Salah Ghabri, France

Daniel Gladwell, UK

Ari Gnanasakthy, USA

Manuel Gomes, UK

Juan Gonzalez, USA

Judith Gout, The Netherlands

Christopher Graham, USA

Carmel Guarnieri, Australia

May Hagiwara, USA

Andrew Hale, Australia

Paul Hanly, Ireland

Fred Hellinger, USA

Peter Hilsenrath, USA

Li Huang, Australia

Rachael Hunter, UK

David Hutton, USA

Sergio Iannazzo, Italy

Ellen Janssen, USA

Michelle Jenks, UK

Ruixuan Jiang, USA

Karissa Johnston, Canada

Claudio Jommi, Italy

Gaurav Jyani, India

Tim Kanters, The Netherlands

Lucy Kanya, UK

Paweł Kawalec, Poland

Stuart Keeping, UK 
Iftekhar Khan, UK

Christopher Knight, UK

Conrad Kobel, Australia

Georgia Kourlaba, Greece

Nicolas Krucien, UK

Karen Lee, Canada

Joseph Levy, USA

Frank Lichtenberg, USA

Luca Lorenzoni, France

Krzysztof Malinowski, Poland

Alexina Mason, UK

Josephine Mauskopf, USA

Stuart Mealing, UK

Sonja Merkesdal, Germany

Alec Miners, UK

Steven Morgan, Canada

David Mott, UK

Aileen Murphy, Ireland

Junice Ng, Singapore

Muhammad Nisar, UK

Daniel Ollendorf, USA

Sara Olofsson, Sweden

Wija Oortwijn, The Netherlands

Gabriela Orsak, USA

Itziar Oyagüez, Spain

George Papadopoulos, Australia

Unchalee Permsuwan, Thailand

Jaime Peters, UK

Laura Pirhonen, Sweden

Daniel Pollard, UK

Xavier Pouwels, The Netherlands
Nigel Rawson, Canada

Fanni Rencz, Hungary

Umberto Restelli, Italy

Luz Reynales-Shigematsu, Mexico

Carlos Rodriguez-Martinez, Colombia

Diego Rosselli, Colombia

Sopany Saing, Australia

Jordana Schmier, USA

Leonie Segal, Australia

Sameera Senanayake, Australia

Soko Setoguchi, USA

Marian Shanahan, Australia

Samantha Slaff, USA

Harry Smolen, USA

William Sullivan, UK

Andrew Sutton, Canada

Alexander Thompson, UK

Dominic Tilden, Australia

Utsana Tonmukayakul, Australia

Dat Tran, Canada

Floortje van Nooten, The Netherlands

Alice Varnava, UK

Jeffrey Voigt, USA

Caroline Watts, Australia

Melanie Whittington, USA

Fernando Wilson, USA

Torbjørn Wisløff, Norway

John Wittenborn, USA

Jipan Xie, USA

Zafar Zafari, USA

Melanie Zeppel, Australia

We also acknowledge the following members of the journal's Honorary Editorial Board, who have reviewed for the journal, contributed articles, and provided guidance on content, policy and processes:

Renée Arnold, USA

James Buchanan, UK

James Chambers, USA

Vakaramoko Diaby, USA

George Dranitsaris, Canada

Stephanie Earnshaw, USA

Flávia Elias, Brazil

Gerardus Frederix, The Netherlands

Emma Frew, UK

Brian Godman, Sweden

Anthony Hatswell, UK

Elisabeth Huynh, Australia

Noam Kirson, USA

Hye-Young Kwon, Republic of Korea

Patrick Lefebvre, Canada

Luís Lizán, Spain

T. Joseph Mattingly II, USA
Nicola McCaffrey, Australia

R. Brett McQueen, USA

Richard Norman, Australia

Alison Pearce, Australia

Paolo Pertile, Italy

Pedro Plans-Rubió, Spain

Shankar Prinja, India

Daniela Roggeri, Italy

Jeffrey Round, Canada

Christopher Sampson, UK

Asrul Shafie, Malaysia

Jonathan Tosh, UK

Haitham Tuffaha, Australia

Sabine Vogler, Austria

Pei Wang, Republic of China

Beth Woods, UK 
Last but not least, we thank our readers for supporting PharmacoEconomics - Open. We hope you have found value in the articles published in 2019, and look forward to providing you with more quality content in 2020.

With best wishes

Timothy Wrightson and Christopher Carswell

Co-Editors in Chief

PharmacoEconomics - Open
Open Access This article is distributed under the terms of the Creative Commons Attribution-NonCommercial 4.0 International License (http://creativecommons.org/licenses/by-nc/4.0/), which permits any noncommercial use, distribution, and reproduction in any medium, provided you give appropriate credit to the original author(s) and the source, provide a link to the Creative Commons license, and indicate if changes were made. 